\title{
Narcolepsy an often missed diagnosis: First documented case from India
}

\begin{abstract}
Sir,
Narcolepsy is an uncommon but important cause of excessive daytime sleepiness, sleep paralysis, hypnogogic, and hypnopompic hallucinations. ${ }^{[1-3]}$ It can be primary or secondary to stroke, multiple sclerosis, brain tumors, neuro-degenerative disorders, and central nervous system (CNS) infections. ${ }^{[4,5]}$ No report on this condition exists from India. We describe probably the first documented case of narcolepsy diagnosed on the basis of clinical history and sleep studies.
\end{abstract}

A 30-year-old male, presented with history of excessive daytime sleepiness since childhood, and sudden episodic weakness of all four limbs with emotional excitement (on multiple occasions, occurring on excessive laughter or fear). He also gave history of taking regular naps since childhood that persisted throughout his college days. However, the tendency for napping had increased for the last three years with history of dozing off while driving. He gave history of falling asleep while sitting, reading, watching television, as a passenger in a car, and recently even while talking to someone. There was history of weakness, which was precipitated by emotional triggers such as laughter and anger, and sudden loud sound such as a loud horn, following which he was unable to press the clutch/break (if he was driving). He also admitted to stammering speech during excitement. He gave history of excessive dreaming during the naps. There was no history of snoring, choking, frequent awakenings, abnormal behavior, morning headache, or increased leg movements in sleep. He was not a known hypertensive or diabetic and was not on any regular medicines. There was no family history of a similar illness. He denied history of previous neurological disease/head trauma.

On examination, he was a well built, nonobese person with normal general physical examination, height $167 \mathrm{~cm}$, weight $75 \mathrm{~kg}$, and body mass index (BMI) 26.5 . Systemic examination including nervous system, revealed no abnormality.

Routineblood biochemistry and hematological investigations 
were normal. Thyroid profile was normal. Magnetic resonance imaging (MRI) and electroencephalography (EEG) were normal. He was subjected to an overnight polysomnography (PSG), ${ }^{[6]}$ which revealed shortened sleep latency $(10 \mathrm{mts})$, early rapid eye movement sleep (REM) onset, with good sleep efficiency and no significant apneas or leg movements. A 4 nap multiple sleep latency test (MSLT) ${ }^{[]]}$was performed the following day (using standardized protocol). The mean sleep latency was 27.5 seconds with presence of REM in two naps. These findings are strongly suggestive of narcolepsy.

CSF examination for hyocretin evaluation was suggested, but the patient was not willing. He was started on modafinil, initially on $100 \mathrm{mg} /$ day then increased to $200 \mathrm{mg} /$ day with fluxotine $20 \mathrm{mg} /$ day and noted significant improvement in daytime sleepiness and cataplexy after four weeks follow-up.

Narcolepsy is a chronic, disabling sleep disorder with a prevalence of approximately 1 in 2,000. Gelineaus first coined the term narcolepsy in 1880. Narcolepsy was defined in International classification of sleep disorders 1997 as "a syndrome characterized by excessive sleepiness, associated with cataplexy and other REM related phenomena such as sleep paralysis and hypnogogic or hypnopompic hallucinations". The age of onset is between 10-50 years but usually before the age of 25 (70-80\% of patients), with no sex predominance. Narcolepsy is thought to be caused by a complex interplay of environmental and genetic factors. ${ }^{[8]}$ Current diagnosis is based primarily on clinical picture, with assistance of sleep recordings and human lymphocyte antigen (HLA) typing. ${ }^{[2,3,5]}$ A MSLT is used to document excessive daytime sleepiness and occurrence of sleep onset REM periods. HLA typing is a supportive test with specificity of DQB10602 positivity being $62 \% \cdot{ }^{[9]}$

Hypocretins/orexins are recently discovered neuropeptides of hypothalamic origin. ${ }^{[10,11]}$ Undetectable cerebrospinal fluid (CSF) hypocretin-1 levels are highly sensitive and specific for narcolepsy, indicating diagnostic utility of CSF-hypocretin-1 measurement. ${ }^{[12]}$ The majority of patients (85-90\%) with narcolepsy - cataplexy have low or undetectable CSF hypocretin-1 Ligand. ${ }^{[13]}$ However, this test is not easily available and our patient was not willing for lumbar puncture.

Excessive daytime sleepness (EDS) is the important symptom of narcolepsy. Cataplexy is found in $60-100 \%$ of patients. The other symptoms are hypnogogic or hypnopompic hallucinations, sleep paralysis, automatic (absent-minded) behavior, and frequent awakening during sleep. ${ }^{[14]}$

Treatment of narcolepsy includes nonpharmacological measures such as frequent structured naps and good sleep hygiene. The pharmacological agents used are stimulants. Modafinil has been evaluated extensively in the treatment of EDS of narcolepsy. It was used as first-line therapy for EDS. ${ }^{[15,16]}$ Sodium oxybate has been recommended for use in narcolepsy. It enhances alertness with significant benefit for cataplexy. ${ }^{[17]}$ The exact mechanism of action is unknown. Other agents used for cataplexy include antidepressants (tri-cyclic and SSRIs). ${ }^{[18]}$

Thus, the patient described clinically meets the criteria of narcolepsy with positive history of excessive sleepiness, cataplexy, excessive dreaming, and disturbed sleep. His overnight PSG with MSLT were suggestive of narcolepsy. This is the first case being reported from India.

This entity requires a high index of suspicion and awareness so that these cases can be identified early and appropriately managed. This will prevent delay in diagnosis and initiation of treatment resulting in improved quality of life. There is a need for a multicenter study to define the frequency of narcolepsy in India. If the frequency is really rare the possible reasons need to be studied. It may be related to the frequency distribution of HLA in the country.

M. Bhatia, M. A. Arif ${ }^{1}$

Sleep Medicine ${ }^{1}$, Clinical Neurophysiology, Sir Ganga Ram Hospital, New Delhi - 110 060, India.

E-mail: Manvirbhatia1@yahoo.com

DOI: $10.4103 / 0028-3886.55588$

\section{References}

1. Bassetti C, Aldrich M. Narcolepsy. Neurol Clin 1996;14:545-71.

2. Nishino S, Okura M, Mignot M. Narcolepsy: Genetic predisposition and neuropharmacological mechanisms. Sleep Med Rev 2000;4:57-99.

3. Victor M, Ropper AH. Principles of neurology. New York: MeGraw-Hill Medical Publishing Division; 2001. p. 421-4.

4. Scammell TE. The neurobiology, diagnosis and treatment of Narcolepsy. Ann Neurol 2003;53:154-6.

5. Aldrich MS. Narcolepsy. Neurology 1992;42:34-43.

6. McGregor P, Weitzman ED, Pollack CP, Polysomnographic recording techniques used for diagnosis of sleep disorders in a sleep disorder center. Am J EEG Technol 1978;18:107-32.

7. Aldrich MS, Chervin RD, Malow BA. Value of the multiple sleep latency test (MSLT) for the diagnosis of narcolepsy. Sleep 1997;20:620-9.

8. Mignot E. Genetic and familial aspects of narcolepsy. Neurology 1998;50:S16-22.

9. Mignot E, Young T, Lin L, Finn L, Palta M. Reduction of REM sleep latency associated with HLA-DQBI *0602 in normal adults. Lancet 1998;351-727.

10. de Lecea L, Kilduff TS, Peyron C, Gao X, Foye PE, Danielson PE, et al. The Hypocretins: Hypothalamus-specific peptides with neuroexcitatory activity. Proc Nalt Acad Sci USA 1998;95:322-7.

11. Sakurai T, Amemiya A, Ishil M, Matsuzaki I, Chemelli RM, Tanaka H, et al. Orexins and orexin receptors: A family of hypothalamic neuropeptides and G protein-coupled receptors that regulate feeding behavior. Cell 1998;92:573-85.

12. Nishino S, Ripley B, Overeem S, Lammers GL, Mignot E. Hypocretin (Orexin) deficiency in human narcolepsy. Lancet 2000;355:39-40. 
13. Ripley B, Overeem S, Fujiki N, Nevsimalova S, Uchino M, Yesavage J, et al. CSF hypocretin/orexin levels in narcolepsy and other neurological conditions. Neurology 2001;57:2253-8.

14. Honda Y. Clinical features of narcolepsy: Japanese experiences. In: Honda Y, Juji T, editors. HLA in narcolepsy. Berlin: Springer-Verlag; 1988. p. 24-57.

15. US Modafinil in Narcolepsy Multicenter Study Group. Randomized trial of modafinil as a treatment for the excessive daytime somnolence of narcolepsy. Neurology 2000;54:1166:75.

16. US Modafinil in Narcolepsy Multicenter Study Group. Randomised trial of modafinil for the treatment of pathological somnolence in narcolepsy. Ann Neurol 1998;43:88-97.

17. Tunnicliff G, Cash CD. Gamma-hydroxybutyrate: Molecular, functional and clinical aspects. New York: Taylor and Francis, Inc.; 2002

18. Nishino S, Mignot E. Pharmacological aspects of human and canine narcolepsy. Prog Neurobiol 1997;52:27-78.

Accepted on 28-07-2009 